\title{
Excitation of Mars polar motion
}

\author{
V. Dehant, O. de Viron, O. Karatekin, and T. Van Hoolst
}

Royal Observatory of Belgium, Avenue Circulaire, 3, 1180 Brussels, Belgium

e-mail:v.dehant@oma.be

Received 14 July 2005 / Accepted 9 September 2005

ABSTRACT

In this paper, computations of Mars' polar motion are presented for Mars models with three homogeneous layers as a function of the radius of the inner core and for different excitation possibilities (atmosphere, ice caps, quakes). We estimated the amplitude of the two polar motion normal modes, i.e. the Chandler wobble and the Inner Core wobble, resulting from atmospheric excitation and for a reasonable interval of damping factor values. We show that the signature of the inner core in the polar motion is very small and is unlikely to be detected with the present observational precision. We further investigated the possibility of exciting the normal modes of Mars' polar motion through Marsquakes and show that the predicted quake moments are not large enough to excite polar motion to an observable level.

Key words. planets and satellites: general

\section{Introduction}

The rotation of Mars is not constant in time and presents irregularities that are mostly associated with the seasonal $\mathrm{CO}_{2}$ mass exchange between Mars' atmosphere and its polar caps. This large mass redistribution, with about a third of the total atmospheric mass being exchanged, also causes variations in the gravity field. By monitoring the orbit of a spacecraft around Mars, such as Mars Global Surveyor or Mars Express, these variations can be deduced and the total mass exchange estimated (Smith et al. 2001; Yoder et al. 2003; Karatekin et al. 2005a). More direct measurements of the mass redistribution can be obtained from determining the ice coverage and height with laser altimetry (Smith et al. 1999) and spectrometry (Litvak et al. 2004). These seasonal changes induce variations in Mars' rotation speed, as well as in polar motion. The effects of the atmosphere on rotation can best be estimated by using the angular momentum approach. Because of conservation of angular momentum of Mars (solid body and atmosphere), considered isolated, any change in the angular momentum of the atmosphere is associated with an opposite change in the angular momentum of the solid body of the planet. The angular momentum of the atmosphere and icecaps can be separated into two parts: a matter term, representing the rigid rotation of the atmosphere and icecaps with the planet, and a wind term accounting for the relative motion of the atmosphere with respect to the surface of the planet.

The direct effect of the atmosphere/ice caps on the rotation has been the topic of numerous studies by Chao \& Rubincam (1990), Cazenave \& Balmino (1981), Defraigne et al. (2000), Van den Acker et al. (2002), Sanchez et al. (2004), Karatekin et al. (2005b). In this study, we focus on the excitation of the polar motion free modes, i.e. the Chandler Wobble $(\mathrm{CW})$ and the Inner core Wobble (ICW), associated with the ellipsoidal shape of the whole planet and the inner core, respectively. The Inner Core wobble can only exist if Mars has a fluid outer core and a solid inner core.

The effect of Marsquakes can also be estimated using the angular momentum approach. Following the approach by Ben-Menahem \& Israel (1970) and Dahlen (1971), the change of angular momentum associated with a quake can be computed from the change in the inertia tensor due to the dislocation, which can be expressed as a function of the fault parameters and of the quake moment.

The goal of this study is to investigate the use of a possible observation of those modes to infer properties of the interior of Mars, such as the inner core radius and the mantle and inner core anelasticity. The amplitudes of the modes, as well as their frequencies, are highly sensitive to the properties of this possible inner core (mostly inertia, anelasticity, and flattening), as shown by Dehant et al. (2003) and Defraigne et al. (2003). In these papers, the eigenfrequencies and the resonance strengths were evaluated in terms of the inner core radius, in the case of a forcing by a harmonic external potential. They found that the inner-core wobble would only reach an observable level for a very large inner core or for an eigenfrequency that is very close to a harmonic forcing frequency.

Except for a few particular frequencies (diurnal, annual, and their lower harmonics), the atmospheric dynamics is, however, far from being harmonic, and the associated excitation is better approximated by a random noise than by a harmonic signal. The theory for computing the response of a three-layer planet to random excitation has been developed by Dehant et al. (2005) for the Earth, who focused on diurnal frequencies and free nutation excitation. Here, we considered long period 
(about one year) forcing and its effects on Mars' polar motion. Mars most likely has a CW period of about 200 days (Van Hoolst et al. 2000; Dehant et al. 2003) between the ter-annual period (223 days) and quadri-annual period (167.25 days). Days in this paper are sidereal Mars days of 88642.7 seconds. The ICW period is also expected to be far from the seasonal periods, except for some particular intervals of values of the inner core radius. In this paper, we therefore consider the non-harmonic excitation of the $\mathrm{CW}$ and ICW by the atmosphere, complementing the previous papers on harmonic forcing.

The paper is organized as follows. In Sect. 2, we present the Mars models used. Section 3 is devoted to adaptation of the equations governing polar motion of a three-layer planet to low frequencies. The Love numbers, used to model the deformation of the planet in response to gravitational forcing and loading, are computed in Sect. 4. In Sect. 5, we examine the response of Mars to a white-noise forcing and present numerical results for the polar motion excitation, using the output of a general circulation model for Mars' atmosphere. In Sect. 6, we adapt the Ben-Menahem \& Israel (1970) computation to Mars and estimate the maximum effect of a Marsquake on polar motion. Conclusions are presented in Sect. 7.

\section{Interior structure models of Mars}

The models used to represent the planet Mars are composed of three homogeneous layers: a solid inner core, a liquid outer core, and a solid mantle. Geodesy provides constraints on the total core radius from the measurements of the moment of inertia. At present, measurements of tidal effects on the orbit of the Mars Global Surveyor spacecraft indicate that Mars has a fluid core (Yoder et al. 2003), but we still have no information on the existence (and dimension) of an inner core. The size of the solid inner core is therefore considered as a parameter of our study, ranging from no inner core at all to a fully solidified core. In order to estimate the sensitivity to the inner core radius, we use inner core radius-dependent Mars parameters, as estimated in Dehant et al. (2003). Dynamically speaking, the three layers are assumed to rotate rigidly, but global deformations of each layer are also estimated by using a linearized elasticity theory, and are represented by Love numbers. Of course, the rigid rotation of the fluid core is not valid stricto sensu. Nevertheless, we can expect that the quasi-rigid motion of the core is a fair approximation, as long as the inner core is not too large, i.e. the core cannot be considered as a thin layer (as long as the boundary layer is only a negligible part of the core volume). Note that we nevertheless investigated inner core sizes ranging from zero to the outer core size, in order to get the limit values, but the hypotheses of our work are clearly not valid anymore for extremely large inner cores.

The formation of the inner core is modelled as follows. First, only iron precipitates to form the inner core, and the concentration of the light elements (considered to be sulfur) increases in the outer core. After reaching the eutectic composition, the inner core grows by "freezing" of the liquid core, i.e. the solidifying material has the same composition as the liquid. Therefore, a second layer forms in the inner core with a different composition and a lower density. Several initial compositions were investigated in Dehant et al. (2003). In this study, we only consider the most commonly accepted one, which is iron with 14 wt\% sulfur (Sohl \& Spohn 1997). With such a composition, the eutectic composition is reached at a radius of $992 \mathrm{~km}$ for a total core radius of $1468 \mathrm{~km}$.

Depending on the radius of the inner core, the interior parameters involved in the expressions for the excitation of the free modes vary. For example, the flattening and moment of inertia of the inner core increase with increasing radius, but also the compositions of the core layers change (see Dehant et al. 2003 , for numerical values). Several of those quantities are displayed in Dehant et al. (2003) and in Fig. 1.

The core flattening $\alpha_{\mathrm{f}}$ is numerically evaluated by assuming hydrostatic equilibrium and ranges between $3.5 \times 10^{-3}$ and $7 \times 10^{-3}$ depending on the inner core radius. The inner core flattening $\alpha_{\mathrm{s}}$ is between $3.5 \times 10^{-3}$ and $4 \times 10^{-3}$.

\section{Polar motion of a three layer Mars}

\subsection{Liouville equations}

The rotation dynamics of a simple Mars model is classically analyzed using the Liouville equation, which is a particular case of the angular momentum budget equation. We use a reference frame rotating with the mantle, with a rotation vector $\boldsymbol{\Omega}$, defined by

$\boldsymbol{\Omega}=\Omega\left(\begin{array}{c}m_{1} \\ m_{2} \\ 1+m_{3}\end{array}\right)$

where $\Omega$ is the mean angular velocity of Mars. Note that the rotation perturbations $m_{i}$ are small with respect to 1 .

The inertia of Mars, $\underline{\bar{I}}$, can be expressed as the inertia of a biaxial ellipsoid perturbed by small deformations:

$\bar{I}=\left(\begin{array}{ccc}A & 0 & 0 \\ 0 & A & 0 \\ 0 & 0 & C\end{array}\right)+\underline{\bar{c}}$

where $A$ is the mean equatorial moment of inertia of Mars and $C$ the polar moment of inertia. The inertia tensors for the outer core and inner core have similar mathematical expressions, with the superscript $f$ for the outer core and $s$ for the inner core.

The incremental part $\underline{\bar{c}}$ is due to the deformation of each layer in response to the forcing potential, to the dislocation associated with a Marsquake, and to change in mass distribution. All the $c_{i j}$ are small with respect to the equilibrium moment of inertia.

The angular momentum of Mars is then given by

$\boldsymbol{H}=\underline{\bar{I}} \boldsymbol{\Omega}+\boldsymbol{h}$.

The additional angular momentum $\boldsymbol{h}$ is associated with the relative angular momentum of the different layers of the whole planet: the inner core $\left(\boldsymbol{h}_{\mathrm{s}}\right)$, the fluid outer core $\left(\boldsymbol{h}_{\mathrm{f}}\right)$, and the atmosphere $\left(\boldsymbol{h}_{\mathrm{a}}\right)$. At the period considered in this study, we can neglect the relative angular momentum associated with the quake itself. All the considered additional angular momenta are small quantities. 

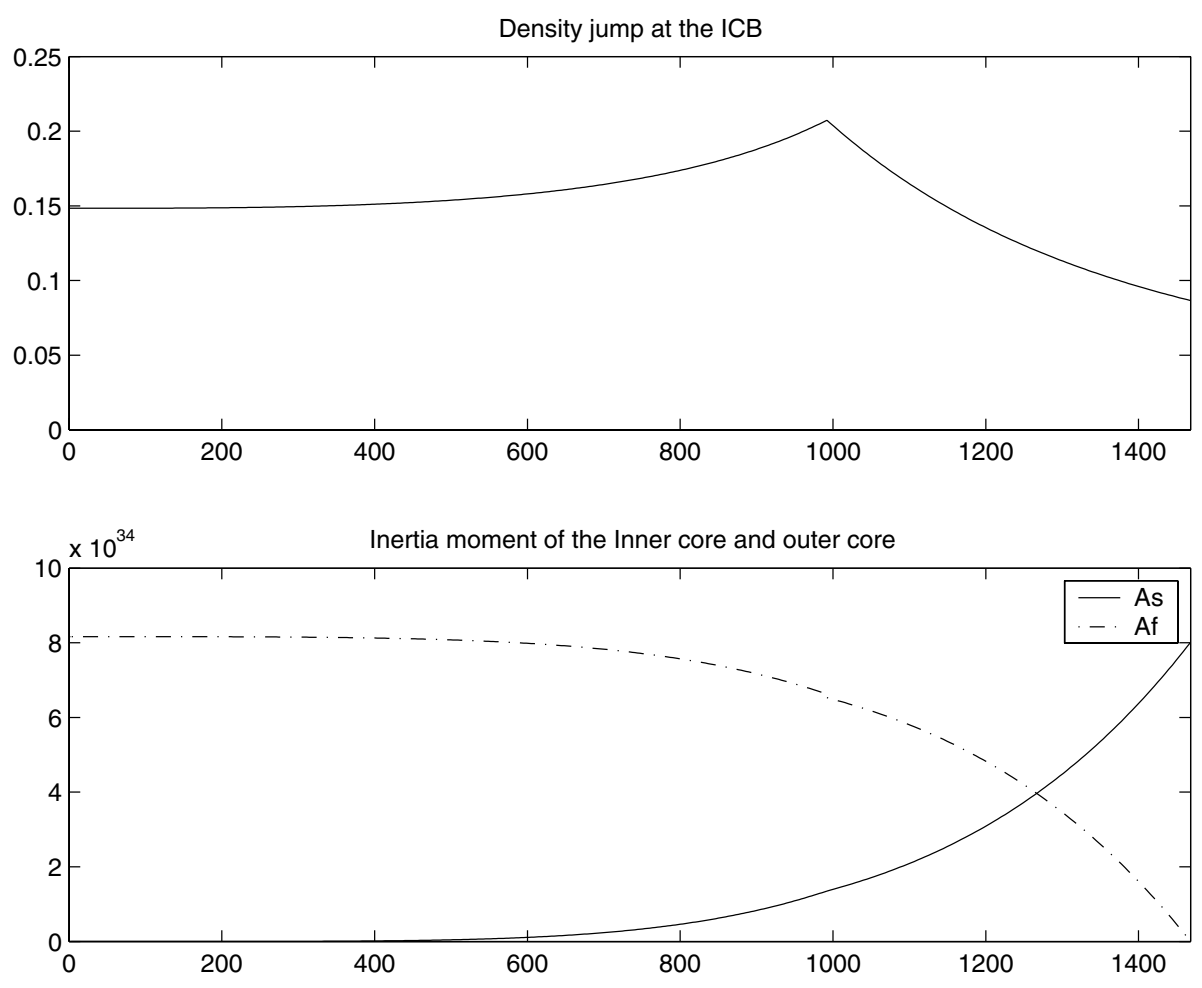

Fig. 1. Numerical values of the density jump (top panel) and of the inertia moment (bottom panel) of the core and inner core as a function of the inner core radius.

As only rigid rotations of the core and inner core are considered in our model, we have:

$\boldsymbol{h}_{\mathrm{s}}=\bar{I}_{\mathrm{s}} \omega_{\mathrm{s}}$
$\boldsymbol{h}_{\mathrm{f}}=\underline{\bar{I}}_{\mathrm{f}} \omega_{\mathrm{f}}$.

With respect to the mantle, the rotation vectors of the inner core and outer core are

$\omega_{\mathrm{s}}=\Omega\left(\begin{array}{c}m_{1}^{\mathrm{s}} \\ m_{2}^{\mathrm{s}} \\ m_{3}^{\mathrm{s}}\end{array}\right)$

$\omega_{\mathrm{f}}=\Omega\left(\begin{array}{c}m_{1}^{\mathrm{f}} \\ m_{2}^{\mathrm{f}} \\ m_{3}^{\mathrm{f}}\end{array}\right)$.

As for the solid planet, the quantities $\bar{c}_{\mathrm{s}}, \bar{c}_{\mathrm{f}}, m_{i}^{\mathrm{f}}$, and $m_{i}^{\mathrm{s}}$ are small. By substituting Eqs. (1)-(7) into the equations expressing the conservation of angular momentum,

$\frac{\mathrm{d} \boldsymbol{H}}{\mathrm{d} t}+\boldsymbol{\Omega} \times \boldsymbol{H}=\boldsymbol{\Gamma}$,

we obtain the Liouville equations, at the first order in the small quantities, for the whole planet. The equatorial part reads

$$
\begin{array}{r}
A \dot{m}-\mathrm{i} \alpha A \Omega m+\dot{c}+\mathrm{i} \Omega c+A_{\mathrm{f}} \dot{m}_{\mathrm{f}}+\mathrm{i} \Omega A_{\mathrm{f}} m_{\mathrm{f}} \\
+A_{\mathrm{s}} \dot{m}_{\mathrm{s}}+\mathrm{i} \Omega A_{\mathrm{s}} m_{\mathrm{s}}+h_{\mathrm{a}}=\frac{\Gamma}{\Omega}
\end{array}
$$

where $\alpha=(C-A) / A$ is the dynamical flattening of the whole planet and $\Gamma$ the complex sum of the first two components of the total torque acting on Mars. Similarly, we use $\alpha_{\mathrm{f}}=\left(C_{\mathrm{f}}-A_{\mathrm{f}}\right) / A_{\mathrm{f}}$ and $\alpha_{\mathrm{s}}=\left(C_{\mathrm{s}}-A_{\mathrm{s}}\right) / A_{\mathrm{s}}$ for the dynamical flattening of the outer core and the inner core, respectively. The complex wobble and inertia increments are defined as:

$$
\begin{aligned}
& m=m_{1}+\mathrm{i} m_{2} \\
& m_{\mathrm{f}}=m_{1}^{\mathrm{f}}+\mathrm{i} m_{2}^{\mathrm{f}} \\
& m_{\mathrm{s}}=m_{1}^{\mathrm{s}}+\mathrm{i} m_{2}^{\mathrm{s}} \\
& c=c_{13}+\mathrm{i} c_{23} \\
& c_{\mathrm{f}}=c_{13}^{\mathrm{f}}+\mathrm{i} c_{23}^{\mathrm{f}} \\
& c_{\mathrm{s}}=c_{13}^{\mathrm{s}}+\mathrm{i} c_{23}^{\mathrm{s}} .
\end{aligned}
$$

In the case of the inner core and outer core, there is no differential angular momentum, as we consider they are rotating rigidly. Consequently, the angular momentum budget equations, at the first order in the small quantities, are given by

$A_{\mathrm{s}} \dot{m}+A_{\mathrm{s}} \dot{m}_{\mathrm{s}}-\mathrm{i} \Omega \alpha_{\mathrm{s}} A_{\mathrm{s}} m+\dot{c}_{\mathrm{s}}+\mathrm{i} \Omega c_{\mathrm{s}}+\mathrm{i} \Omega A_{\mathrm{s}} m_{\mathrm{s}}=\frac{\Gamma_{\mathrm{s}}}{\Omega}$

for the inner core, and by

$A_{\mathrm{f}} \dot{m}+A_{\mathrm{f}} \dot{m}_{\mathrm{f}}-\mathrm{i} \Omega \alpha_{\mathrm{f}} A_{\mathrm{f}} m+\dot{c}_{\mathrm{f}}+\mathrm{i} \Omega c_{\mathrm{f}}+\mathrm{i} \Omega A_{\mathrm{f}} m_{\mathrm{f}}=\frac{\Gamma_{\mathrm{f}}}{\Omega}$

for the liquid outer core, with $\Gamma_{\mathrm{s}}$ the total torque on the inner core and $\Gamma_{\mathrm{f}}$ the total torque on the outer core. For simplicity, the outer core will be noted "core" hereafter.

Equations (9), (16), and (17) are the Liouville equations for a three-layer planet as first derived in Dehant et al. (1993). Here we extend these equations in order to account for the effect of a surface fluid layer. Corrections to these equations, as expressed in Greff-Lefftz et al. (2000) and Greff-Lefftz et al. (2002), are considered as well. 
The effects of the Martian atmosphere on the rotation are included in these equations through the induced changes in three quantities: (1) the relative angular momentum; (2) the inertia tensor; and (3) the interaction torques at the core boundaries. We then derive explicit equations for these quantities.

\subsection{Incremental relative angular momentum}

The relative angular momentum of the external fluid, which is related to the wind in the Martian atmosphere, must be included in the angular momentum budget. This is classically done for the Earth by using the motion (or wind) angular momentum excitation function $\left(\chi_{\mathrm{w}}\right)$, as defined in Munk \& McDonald (1960). The wind excitation function is defined by its relation to the relative atmospheric angular momentum:

$h_{\mathrm{a}}=\alpha A \Omega \chi_{\mathrm{w}}$.

\subsection{Change in the inertia tensor}

As the atmosphere is also included in the "global planet", we have to take it into account when computing the global increment of inertia. Additionally, the loading of the surface fluids induces deformations that change the inertia of the global planet $(c)$, of the core $\left(c_{\mathrm{f}}\right)$, and of the inner core $\left(c_{\mathrm{s}}\right)$. The matter (or pressure) angular momentum excitation function $\left(\chi_{\mathrm{p}}\right)$ is defined by its relation to the inertia change (see Munk \& McDonald 1960). Following Sasao \& Wahr (1981), Dehant et al. (1993), and Dehant et al. (2005), we compute the inertia increments by integration on the deformed volume of the core and of the inner core, and evaluate the topography of the deformed surface from the forcing potential and pressure using the Love number formalism (Greff-Lefftz et al. 2000). We then obtained the following expressions for the inertia change as a function of the rotation variation of the whole planet, of the core, and of the inner core.

$$
\begin{aligned}
c= & \alpha A \frac{k_{0}^{t}}{\kappa} m+\alpha A \frac{\bar{k}_{1}^{t}+\bar{k}_{2}^{t}}{\kappa} m_{\mathrm{f}}+\alpha A \frac{k_{2}^{t}}{\kappa} m_{\mathrm{s}}-3 \alpha A \frac{k_{0}^{t}}{\kappa} \frac{W}{\Omega^{2} a^{2}} \\
& +\alpha A\left(1+\hat{k}_{0}^{t}\right) \chi_{\mathrm{p}} \\
& -\mathrm{i} \alpha_{\mathrm{s}} A_{\mathrm{s}}\left(1-\frac{\rho_{\mathrm{f}}}{\rho_{\mathrm{s}}}\right)\left(1+\left(\frac{a}{c}\right)^{3} \hat{k}_{2}^{t}\right) \theta_{\mathrm{s}}+\Delta c^{\text {quake }} \\
c_{\mathrm{f}}= & \frac{q_{0}}{2}\left(\left(A_{\mathrm{f}}+\frac{\rho_{\mathrm{f}}}{\rho_{\mathrm{s}}} A_{\mathrm{s}}\right) h_{0}^{\mathrm{f}}-A_{\mathrm{s}} h_{0}^{\mathrm{s}} \frac{\rho_{\mathrm{f}}}{\rho_{\mathrm{s}}}\right) m \\
& +\frac{q_{0}}{2}\left(\left(A_{\mathrm{f}}+\frac{\rho_{\mathrm{f}}}{\rho_{\mathrm{s}}} A_{\mathrm{s}}\right) \bar{h}_{1}^{\mathrm{f}}-A_{\mathrm{s}} \bar{h}_{2}^{\mathrm{s}} \frac{\rho_{\mathrm{f}}}{\rho_{\mathrm{s}}}\right) m_{\mathrm{f}} \\
& -A_{\mathrm{s}} h_{\mathrm{s}}^{2} \frac{q_{0}}{2} \frac{\rho_{\mathrm{f}}}{\rho_{\mathrm{s}}} m_{\mathrm{s}} \\
& -3\left(\left(A_{\mathrm{f}}+\frac{\rho_{\mathrm{f}}}{\rho_{\mathrm{s}}} A_{\mathrm{s}}\right) h_{0}^{\mathrm{f}}-A_{\mathrm{s}} h_{0}^{\mathrm{s}} \frac{\rho_{\mathrm{f}}}{\rho_{\mathrm{s}}}\right) \frac{q_{0}}{2} \frac{W}{\Omega^{2} a^{2}} \\
& +\mathrm{i} \alpha_{\mathrm{s}} A_{\mathrm{s}} \frac{\rho_{\mathrm{f}}}{\rho_{\mathrm{s}}}\left(1+x_{\mathrm{s}} h_{2}^{\mathrm{f}}\right) \theta_{\mathrm{s}} \\
& +\left(\left(A_{\mathrm{f}}+\frac{\rho_{\mathrm{f}}}{\rho_{\mathrm{s}}} A_{\mathrm{s}}\right) \hat{h}_{0}^{\mathrm{f}}-A_{\mathrm{s}} \hat{h}_{0}^{\mathrm{s}} \frac{\rho_{\mathrm{f}}}{\rho_{\mathrm{s}}}\right) \alpha \chi_{\mathrm{p}}+\Delta c_{\mathrm{f}}^{\text {quake }}
\end{aligned}
$$

$$
\begin{aligned}
c_{\mathrm{s}}= & A_{\mathrm{s}} h_{0}^{\mathrm{s}} \frac{q_{0}}{2} m+A_{\mathrm{s}} \bar{h}_{2}^{\mathrm{s}} \frac{q_{0}}{2} m_{\mathrm{f}}+A_{\mathrm{s}} h_{2}^{\mathrm{s}} \frac{q_{0}}{2} m_{\mathrm{s}} \\
& -3 A_{\mathrm{s}} h_{0}^{\mathrm{s}} \frac{q_{0}}{2} \frac{W}{\Omega^{2} a^{2}}-\mathrm{i} \alpha_{\mathrm{s}} A_{\mathrm{s}} \theta_{\mathrm{s}}\left(1+x^{\mathrm{s}} \hat{h}_{2}^{\mathrm{s}}\right) \\
& +A_{\mathrm{s}} \hat{h}_{0}^{\mathrm{s}} \alpha \chi_{\mathrm{p}}+\Delta c_{\mathrm{s}}^{\text {quake }}
\end{aligned}
$$

where $\frac{q_{0}}{2}=\Omega^{2} a / g$ is the ratio between the centrifugal and gravitational acceleration at the equator, $g$ the mean surface gravity acceleration; $a, b$, and $c$ are the radii of the total planet, the core, and the inner core, respectively; $\kappa=3(C-A) G /\left(\Omega^{2} r_{\text {surf }}^{5}\right)$ is the fluid Love number; $\rho, \rho_{\mathrm{f}}, \rho_{\mathrm{s}}$ are the (mean) densities of the whole planet, of the core, and of the inner core, respectively; $\Delta \rho=\left(1-\frac{\rho_{\mathrm{f}}}{\rho_{\mathrm{s}}}\right)$, and $x_{\mathrm{s}}=(4 \pi G / 5) \Delta \rho c / g$. The Love numbers $\left(k_{0}^{t}\right.$ and $\left.h_{0}^{t}\right)$ are the classical Love numbers, expressing the deformation and mass redistribution of the planet in response to a potential. Love numbers with a bar express the deformation in response to a pressure, while the same symbols with a hat express the response to a surface load (pressure + gravitational attraction). For the inner core and the core, these equations are written with superscripts $\mathrm{s}$ and $\mathrm{f}$ instead of $t$, respectively. Subscripts 2 and 1 , instead of 0 , indicate that the forcing is applied at the Inner Core Boundary (ICB) and Core Mantle Boundary (CMB), respectively. For the effect of a load at the ICB on the core inertia, we need to account for the effect of both the deformation of the ICB and the CMB. After some algebra, it can be shown that it is equivalent to having a Love number $h_{2}^{\prime \text { f }}$ equal to

$\frac{\rho_{\mathrm{s}}}{\rho_{\mathrm{f}}} \hat{h}_{2}^{\mathrm{f}}+\frac{A_{\mathrm{s}}}{A_{\mathrm{f}}} \hat{h}_{2}^{\mathrm{s}} \frac{c}{b}$

We also allow for a tilt of the inner core with respect to the mantle. The angle of this tilt is given by $\theta_{\mathrm{s}}$. This quantity is linked to the inner core wobble by $\Omega m_{\mathrm{s}}=\dot{\theta}_{\mathrm{s}}$.

In Eqs. (19)-(21), the effects of Marsquakes on the products of inertia are noted as $\Delta c^{\text {quake }}, \Delta c_{\mathrm{f}}^{\text {quake }}$, and $\Delta c_{\mathrm{s}}^{\text {quake }}$ for the whole planet, the outer core, and the inner core, respectively. An excitation due to a Marsquake is totally different with respect to the atmospheric excitation both in terms of dynamics and frequency behavior. Consequently, the effects of Marsquakes will be considered separately in the last section of this paper.

\subsection{Change in the interaction torques}

The interaction torque $\boldsymbol{\Gamma}$ on the outer core is estimated from the local torques

$$
\begin{aligned}
\boldsymbol{\Gamma}= & \int_{V \mathrm{oc}} \boldsymbol{r} \wedge \boldsymbol{\nabla} P_{\mathrm{f}} \mathrm{d} V+\int_{V \mathrm{oc}} \boldsymbol{r} \wedge \rho_{\mathrm{f}} \boldsymbol{\nabla} \phi \mathrm{d} V \\
& +\int_{V \mathrm{oc}} \boldsymbol{r} \wedge \rho_{\mathrm{f}} \boldsymbol{\nabla} W \mathrm{~d} V .
\end{aligned}
$$

Using the Navier-Stokes equation in absence of nonconservative forces, it is possible to express the pressure on the fluid $\left(P_{\mathrm{f}}\right)$ as a function of the acceleration potential $(\pi)$ (see Dehant et al. 1993), the centrifugal potential $(\psi)$, 
the gravitational forcing potential $(W)$, and the mass redistribution potential $(\phi)$. After some basic algebra, we obtain:

$\boldsymbol{\Gamma}_{\mathrm{f}}=\int_{V o c} \rho_{\mathrm{f}} \boldsymbol{r} \wedge \boldsymbol{\nabla}(\pi+\psi) \mathrm{d} V$.

The torque on the inner core and the interpretation must be performed over the volume of the inner core instead of over the volume of the outer core. It is computed from an equation similar to Eq. (23), with the density $\rho_{\mathrm{s}}$ rather than $\rho_{\mathrm{f}}$. After substituting the expression for the fluid pressure, we get

$$
\begin{aligned}
\boldsymbol{\Gamma}_{\mathrm{s}}= & \int_{V \text { ic }} \rho_{\mathrm{f}} \boldsymbol{r} \wedge \boldsymbol{\nabla}(\pi+\psi) \mathrm{d} V \\
& +\int_{V \text { ic }}\left(\rho_{\mathrm{s}}-\rho_{\mathrm{f}}\right) \boldsymbol{r} \wedge \boldsymbol{\nabla}(\phi+W) \mathrm{d} V .
\end{aligned}
$$

As in the case of the outer core, the first term is not affected by the presence of a surface fluid. But in the second term, the mass of the surface fluid has to be included in the forcing potential $W$, and the resulting deformation has to be included in the mass redistribution potential $\phi$. This last effect is at the second order, so will be neglected. After some (heavy) algebra, we obtain the following final expression for the torque on the inner core:

$$
\begin{aligned}
\Gamma_{\mathrm{s}}= & -3 \mathrm{i} A_{\mathrm{s}}\left(\alpha_{\mathrm{s}}\left(1+k_{0}^{\mathrm{s}}\right)\left(1-\frac{\rho_{\mathrm{f}}}{\rho_{\mathrm{s}}}\right)+\frac{q_{0}}{2} h_{0}^{\mathrm{s}} \frac{\rho_{\mathrm{f}}}{\rho_{\mathrm{s}}}\right) \frac{W}{a^{2}} \\
& -\mathrm{i} A_{\mathrm{s}} \Omega^{2} m\left(\alpha_{\mathrm{s}}-\frac{q_{0}}{2} h_{0}^{\mathrm{s}}\right)\left(\frac{\rho_{\mathrm{f}}}{\rho_{\mathrm{s}}}\left(1+k_{2}^{\mathrm{s}}\right)-k_{2}^{\mathrm{s}}\right) \\
& +\mathrm{i} A_{\mathrm{s}} \Omega^{2} m_{\mathrm{f}}\left(\frac{\rho_{\mathrm{f}}}{\rho_{\mathrm{s}}} \frac{q_{0}}{2} \bar{h}_{2}^{\mathrm{s}}-\alpha_{\mathrm{s}}\left(\frac{\rho_{\mathrm{f}}}{\rho_{\mathrm{s}}}\left(1+\bar{k}_{2}^{\mathrm{s}}\right)-\bar{k}_{2}^{\mathrm{s}}\right)\right) \\
& +\mathrm{i} A_{\mathrm{s}} \Omega^{2} m_{\mathrm{s}}\left(\frac{\rho_{\mathrm{f}}}{\rho_{\mathrm{s}}} \frac{q_{0}}{2} h_{2}^{\mathrm{s}}-\alpha_{\mathrm{s}}\left(1-\frac{\rho_{\mathrm{f}}}{\rho_{\mathrm{s}}}\right) k_{2}^{\mathrm{s}}\right) \\
& -\frac{5}{3} \Omega^{2}\left(1-\frac{\rho_{\mathrm{f}}}{\rho_{\mathrm{s}}}\right) \alpha_{\mathrm{s}} A_{\mathrm{s}}\left(1+\hat{k}_{0}^{\mathrm{s}}\right) \kappa \chi_{\mathrm{p}} .
\end{aligned}
$$

\subsection{The modified Liouville equations}

By substituting the expressions of the increments of inertia and of the interaction torques into Eqs. (9), (16), and (17), the angular momentum budget equation can be expressed in terms of the rotation vector of the mantle, the core, and the inner core, as well as in terms of the forcing potential $(W)$ and the atmospheric excitation functions $\left(\chi_{\mathrm{p}}\right.$ and $\left.\chi_{\mathrm{w}}\right)$.

The final form of the equation for the global planet is:

$$
\begin{aligned}
\mathrm{i} \Omega m(\sigma & \left.-\Omega \alpha+\alpha \frac{k_{0}^{t}}{\kappa}(\Omega+\sigma)\right) \\
& +\mathrm{i} \Omega m_{\mathrm{f}}(\Omega+\sigma)\left(\frac{A_{\mathrm{f}}}{A}+\alpha\left(\frac{\bar{k}_{2}^{t}}{\kappa}+\frac{\bar{k}_{1}^{t}}{\kappa}\right)\right) \\
& +\mathrm{i} \Omega m_{\mathrm{s}}(\Omega+\sigma)\left(\frac{A_{\mathrm{s}}}{A}+\alpha \frac{k_{2}^{t}}{\kappa}\right) \\
& +\alpha_{\mathrm{s}} \Omega \frac{A_{\mathrm{s}}}{A} \theta(\Omega+\sigma)\left(1-\frac{\rho_{\mathrm{f}}}{\rho_{\mathrm{s}}}\right)\left(1+\hat{k}_{2}^{t}\right) \\
= & -\mathrm{i} \alpha \Omega(\Omega+\sigma)\left(\chi_{\mathrm{P}}\left(1+\hat{k}_{0}^{t}\right)+\chi_{\mathrm{W}}\right) \\
& -3 \mathrm{i} \alpha\left(\Omega-(\Omega+\sigma) \frac{k_{0}^{t}}{\kappa}\right) \frac{W}{a^{2} \Omega} .
\end{aligned}
$$

Similarly, the final form of the equation for the fluid core is:

$$
\begin{aligned}
\mathrm{i} \sigma \Omega m+ & \mathrm{i} \Omega \sigma m \frac{q_{0}}{2}\left(h_{0}^{\mathrm{f}}+\frac{A_{\mathrm{s}}}{A_{\mathrm{f}}} \frac{\rho_{\mathrm{f}}}{\rho_{\mathrm{s}}}\left(h_{0}^{\mathrm{f}}-h_{0}^{\mathrm{s}}\right)\right) \\
& +\mathrm{i} \Omega m_{\mathrm{f}}\left(\Omega \alpha_{\mathrm{f}}+(\Omega+\sigma)\right) \\
& +\mathrm{i} \Omega \sigma m_{\mathrm{f}} \frac{q_{0}}{2}\left(\bar{h}_{1}^{\mathrm{f}}+\frac{A_{\mathrm{s}}}{A_{\mathrm{f}}} \frac{\rho_{\mathrm{f}}}{\rho_{\mathrm{s}}}\left(\bar{h}_{1}^{\mathrm{f}}-\bar{h}_{2}^{\mathrm{s}}\right)\right) \\
& -\mathrm{i} \Omega \sigma m_{\mathrm{s}} \frac{A_{\mathrm{s}}}{A_{\mathrm{f}}} \frac{\rho_{\mathrm{f}}}{\rho_{\mathrm{s}}} \frac{q_{0}}{2} h_{2}^{\mathrm{s}}-\alpha_{\mathrm{s}} \Omega \sigma \theta \frac{\rho_{\mathrm{f}}}{\rho_{\mathrm{s}}} \frac{A_{\mathrm{s}}}{A_{\mathrm{f}}}\left(1+x^{\mathrm{s}} h_{2}^{\prime \mathrm{f}}\right) \\
= & 3 \mathrm{i} \frac{q_{0}}{2} \sigma\left(h_{0}^{\mathrm{f}}+\frac{A_{\mathrm{s}}}{A_{\mathrm{f}}} \frac{\rho_{\mathrm{f}}}{\rho_{\mathrm{s}}}\left(h_{0}^{\mathrm{f}}-h_{0}^{\mathrm{s}}\right)\right) \frac{W}{\Omega a^{2}} \\
& -\mathrm{i} \alpha \Omega \sigma \chi_{\mathrm{P}}\left(\hat{h}_{0}^{\mathrm{f}}+\frac{A_{\mathrm{s}}}{A_{\mathrm{f}}} \frac{\rho_{\mathrm{f}}}{\rho_{\mathrm{s}}}\left(\hat{h}_{0}^{\mathrm{f}}-\hat{h}_{0}^{\mathrm{s}}\right)\right)
\end{aligned}
$$

and we obtain, for the inner core:

$$
\begin{aligned}
\mathrm{i} \Omega m(\sigma & \left.-\Omega \alpha_{\mathrm{s}}\left(1-\frac{\rho_{\mathrm{f}}}{\rho_{\mathrm{s}}}\right)\right)+\mathrm{i} \Omega m \frac{q_{0}}{2} h_{2}^{t}\left(\sigma+\Omega\left(1-\frac{\rho_{\mathrm{f}}}{\rho_{\mathrm{s}}}\right)\right) \\
& -\mathrm{i} \alpha_{\mathrm{s}} \Omega^{2} m\left(1-\frac{\rho_{\mathrm{f}}}{\rho_{\mathrm{s}}}\right) k_{2}^{t} \\
& +\mathrm{i} \alpha_{\mathrm{s}} \Omega^{2} m_{\mathrm{f}} \frac{\rho_{\mathrm{f}}}{\rho_{\mathrm{s}}}+\mathrm{i} \Omega m_{\mathrm{f}} \frac{q_{0}}{2} \bar{h}_{2}^{\mathrm{s}}\left(\sigma+\Omega\left(1-\frac{\rho_{\mathrm{f}}}{\rho_{\mathrm{s}}}\right)\right) \\
& -\mathrm{i} \alpha_{\mathrm{s}} \Omega^{2} m_{\mathrm{f}}\left(1-\frac{\rho_{\mathrm{f}}}{\rho_{\mathrm{s}}}\right) \bar{k}_{2}^{\mathrm{s}} \\
& +\mathrm{i} \Omega(\sigma+\Omega) m_{\mathrm{s}}+\mathrm{i} \Omega m_{\mathrm{s}} \frac{q_{0}}{2} h_{2}^{\mathrm{s}}\left(\sigma+\Omega\left(1-\frac{\rho_{\mathrm{f}}}{\rho_{\mathrm{s}}}\right)\right) \\
& -\mathrm{i} \alpha_{\mathrm{s}} \Omega^{2} m_{\mathrm{s}}\left(1-\frac{\rho_{\mathrm{f}}}{\rho_{\mathrm{s}}}\right) k_{2}^{\mathrm{s}} \\
& +\alpha_{\mathrm{s}} \Omega \theta\left(\sigma+\Omega\left(1-\frac{\rho_{\mathrm{f}}}{\rho_{\mathrm{s}}}\right)\left(1+x^{\mathrm{s}} \hat{h}_{2}^{\mathrm{s}}\right)\right) \\
& +\alpha_{\mathrm{s}} \Omega^{2} k_{2}^{\mathrm{s}} \theta\left(1-\frac{\rho_{\mathrm{f}}}{\rho_{\mathrm{s}}}\right) \\
= & -\mathrm{i} \alpha \Omega \chi \mathrm{P} \bar{h}_{0}^{\mathrm{s}}\left(\sigma+\Omega\left(1-\frac{\rho_{\mathrm{f}}}{\rho_{\mathrm{s}}}\right)\right) \\
& -3 \mathrm{i} \frac{W}{a^{2}}\left(\left(1-\frac{\rho_{\mathrm{f}}}{\rho_{\mathrm{s}}}\right) \alpha_{\mathrm{s}}\left(1+k_{0}^{\mathrm{s}}\right)\right. \\
& \frac{q_{0}}{2 \Omega} h_{0}^{\mathrm{s}}\left(\sigma+\Omega\left(1-\frac{\rho_{\mathrm{f}}}{\rho_{\mathrm{s}}}\right)\right) .
\end{aligned}
$$

\subsection{The motion equations with the low frequency approximation}

For the study of polar motion, we limit ourselves to a lowfrequency approximation of Eqs. (27)-(29) $(|\sigma| \ll \Omega)$. The system of equations can then be written as

$$
\begin{gathered}
\left(\begin{array}{ccc}
-\lambda_{1}+\sigma \frac{A_{\mathrm{f}}}{A} \Omega \frac{A_{\mathrm{s}}}{A} \Omega\left(\sigma+\lambda_{t}\right) & \\
\sigma & \Omega & 0 \\
\sigma+\lambda_{x} & 0 & \Omega\left(\sigma-\lambda_{2}\right)
\end{array}\right)\left(\begin{array}{c}
m \\
m_{\mathrm{f}} \\
m_{\mathrm{s}}
\end{array}\right)= \\
\left(\begin{array}{c}
-3 \alpha\left(1-\frac{k_{o}^{t}}{\kappa}\right) \frac{W}{a^{2}}-\alpha \Omega^{2}\left(\left(1+\hat{k}_{0}^{t}\right) \chi_{\mathrm{P}}+\chi_{\mathrm{W}}\right) \\
3 \sigma\left(a_{3}+\frac{A_{\mathrm{s}}}{A_{\mathrm{f}}} a_{4}\right) \frac{W}{\Omega a^{2}}-\Omega \sigma\left(a_{5}+\frac{A_{\mathrm{s}}}{A_{\mathrm{f}}} a_{6}\right) \chi_{\mathrm{P}} \\
3 a_{2} \frac{W}{a^{2}}+\Omega^{2} a_{1} \chi_{\mathrm{P}}
\end{array}\right),
\end{gathered}
$$


where we have introduced the following constants

$$
\begin{aligned}
& a_{1}=-\alpha \bar{h}_{0}^{\mathrm{s}}\left(1-\frac{\rho_{\mathrm{f}}}{\rho_{\mathrm{s}}}\right) \\
& a_{2}=-\left(1-\frac{\rho_{\mathrm{f}}}{\rho_{\mathrm{s}}}\right)\left(\alpha_{\mathrm{s}}\left(1+k_{0}^{\mathrm{s}}\right)-\frac{q_{0}}{2} h_{0}^{\mathrm{s}}\right) \\
& a_{3}=\frac{q_{0}}{2} h_{0}^{\mathrm{f}} \\
& a_{4}=\frac{q_{0}}{2} \frac{\rho_{\mathrm{f}}}{\rho_{\mathrm{s}}}\left(h_{0}^{\mathrm{f}}-h_{0}^{\mathrm{s}}\right) \\
& a_{5}=\alpha \hat{h}_{0}^{\mathrm{f}} \\
& a_{6}=\frac{q_{0}}{2} \frac{\rho_{\mathrm{f}}}{\rho_{\mathrm{s}}}\left(\hat{h}_{0}^{\mathrm{f}}-\bar{h}_{0}^{\mathrm{s}}\right) \\
& \lambda_{1}=\Omega \alpha\left(1-\frac{k_{0}^{t}}{\kappa}\right) \\
& \lambda_{2}=\Omega \alpha_{\mathrm{s}} \frac{\rho_{\mathrm{s}}-\rho_{\mathrm{f}}}{\rho_{\mathrm{s}}}\left(1+k_{2}^{\mathrm{s}}\right)\left(1+x^{\mathrm{s}} \hat{h}_{2}^{\mathrm{s}}\right) \\
& \lambda_{x}=\Omega\left(\alpha \frac{k_{2}^{t}}{\kappa}-\frac{A_{\mathrm{s}}}{A} \alpha_{\mathrm{s}} \frac{\rho_{\mathrm{s}}-\rho_{\mathrm{f}}}{\rho_{\mathrm{s}}}\right) \\
& \lambda_{t}=-\alpha_{\mathrm{s}} \Omega \frac{A_{\mathrm{s}}}{A} \frac{\rho_{\mathrm{s}}-\rho_{\mathrm{f}}}{\rho_{\mathrm{s}}} .
\end{aligned}
$$

The homogeneous system (30) leads to two eigenmodes, the Chandler Wobble (CW) and the Inner Core Wobble (ICW).

Unlike what was done in Dehant et al. (1993) and Dehant et al. (2005), we cannot neglect the terms in $A_{\mathrm{s}}^{2}$ with respect to $A^{2}$ in the solutions, as $A_{\mathrm{s}}$ can become as large as $10 \%$ of $A$ for large inner core radii. But we cannot find analytical expressions for the free mode periods without making some kind of approximations. The one that seemed the most efficient to us was to consider that $\lambda_{x}=-\lambda_{2}$. The associated error is mostly related to deformation and is smaller than $1 \%$. This approximation leads to a very elegant analytical solution, where the terms in $A_{\mathrm{f}}$, which are small for a large inner core, and the terms in $A_{\mathrm{s}}$, which are small for a small inner core, are completely decoupled. The frequencies of the 2 modes are then given by

$$
\begin{aligned}
\sigma_{\mathrm{CW}} & =\frac{A \lambda_{1}+A_{\mathrm{s}} \lambda_{t}}{A_{m}}, \\
\sigma_{\mathrm{ICW}} & =\lambda_{2},
\end{aligned}
$$

and the solution can be expressed as

$$
\begin{aligned}
m= & \frac{A_{\mathrm{f}}}{A_{m}} S_{\mathrm{TI}} f+\frac{A_{\mathrm{s}}}{A_{m}} T_{\mathrm{TI}} f \\
& +\left(\frac{A}{A_{m}} R_{\mathrm{CW}}+\frac{A_{\mathrm{f}}}{A_{m}} S_{\mathrm{CW}}+\frac{A_{\mathrm{s}}}{A_{m}} T_{\mathrm{CW}}\right) \frac{f}{\sigma-\sigma_{\mathrm{CW}}} \\
& +\frac{A_{\mathrm{s}}}{A_{m}} \frac{f T_{\mathrm{ICW}}}{\sigma-\sigma_{\mathrm{ICW}}},
\end{aligned}
$$

where $f$ is the excitation function, which could be either the external potential $\left(\frac{3 W}{\Omega^{2} a^{2}}\right)$, the wind $\left(\chi_{\mathrm{W}}\right)$, the matter $\left(\chi_{\mathrm{P}}\right)$ excitation functions, or can be associated with the change of inertia products induced by the quakes. The analytical expressions for the resonance strength parameters $\left(S_{\mathrm{TI}}, T_{\mathrm{TI}}, R_{\mathrm{CW}}, S_{\mathrm{CW}}, T_{\mathrm{CW}}\right.$, and $\left.T_{\mathrm{ICW}}\right)$ are given in Table 1.
Table 1. Contribution of the external forcing, and the matter and wind excitations to the resonance strength parameters.

\begin{tabular}{ccccccc}
\hline \hline & $R_{\mathrm{CW}}$ & $S_{\mathrm{TI}}$ & $S_{\mathrm{CW}}$ & $T_{\mathrm{TI}}$ & $T_{\mathrm{CW}}$ & $T_{\mathrm{IC}}$ \\
\hline$\frac{3 W}{\Omega^{2} a^{2}}$ & $-\alpha \Omega\left(1-\frac{k_{0}^{t}}{\kappa}\right)$ & $-a_{3}$ & $-a_{3} \sigma_{\mathrm{CW}}$ & $a_{4}$ & $-\Omega a_{2} \frac{\sigma_{\mathrm{CW}}+\lambda_{t}}{\sigma_{\mathrm{CW}}-\sigma_{\mathrm{IC}}}$ & $\Omega a_{2} \frac{\lambda_{2}+\lambda_{t}}{\sigma_{\mathrm{CW}}-\sigma_{\mathrm{IC}}}$ \\
$\chi_{\mathrm{P}}$ & $-\alpha \Omega\left(1+\hat{k}_{0}^{t}\right)$ & $a_{5}$ & $a_{5} \sigma_{\mathrm{CW}}$ & $a_{6}$ & $-\Omega a_{1} \frac{\sigma_{\mathrm{CW}}+\lambda_{t}}{\sigma_{\mathrm{CW}}-\sigma_{\mathrm{IC}}}$ & $\Omega a_{1} \frac{\lambda_{2}+\lambda_{t}}{\sigma_{\mathrm{CW}}-\sigma_{\mathrm{IC}}}$ \\
$\chi_{\mathrm{W}}$ & $-\alpha \Omega$ & 0 & 0 & 0 & 0 & 0 \\
\hline
\end{tabular}

\section{Love numbers and inner core radius}

In this study, we limit ourselves to the linear theory of elasticity, and the deformations are accounted for by the Love numbers. In order to study the sensitivity to the inner core radius of the $\mathrm{CW}$ and ICW response, we calculated the Love numbers for a set of Mars models with different inner core radii. The details about the analytical expressions of these Love numbers are given in Dehant et al. (2003) and Dehant et al. (2005).

In Fig. 1, we show the density jump and the inertia moments as a function of the inner core radius. In Fig. 2, we display the relevant Love numbers for models with an inner core radius between 0 and the core radius. As expected, only the Love numbers corresponding to deformation of the inner core show an important variation with the inner core radius.

\section{Excitation of the normal modes by the atmosphere and ice caps}

In Sect. 3, we developed the equations for the rotation of a three-layer Mars in response to an excitation by either an external potential or a superficial fluid layer. The corresponding excitation functions, $\chi_{\mathrm{P}}$ and $\chi_{\mathrm{W}}$, associated respectively with the matter and the motion terms of the angular momentum, can be computed easily from the output of a Martian atmosphere Global Circulation Model (GCM).

The general circulation of Mars' atmosphere shows a large annual and semi-annual signal, in addition to the diurnal cycle. From previous studies (see for instance Van den Acker et al. 2002), it is expected that the polar motion of Mars, both the seasonal components and those associated with the normal modes, will be small at the level of the decimeter or the meter at the planet's surface, whereas it is at the ten meter level at the Earth's surface.

As shown by Dehant et al. (2003), the Chandler wobble period does not vary much with the inner core radius and has a typical value of 200 days. The Inner Core wobble period does not vary much for an inner core radius smaller than about a third of the core radius. If the eutectic composition is not reached at a third of the core radius, which is the case for low sulfur concentration, the inner core wobble period decreases noticeably with the radius. After the eutectic composition, the ICW period increases with the inner core radius at a ratio on the order of 1 day per kilometer. In our study of a Mars model with a $14 \mathrm{wt} \%$ sulfur content, the ICW has a period close to 550 days before the eutectic composition (corresponding to an inner core radius of about $992 \mathrm{~km}$ ) and reaches a period of 1000 days for a very large inner core. 

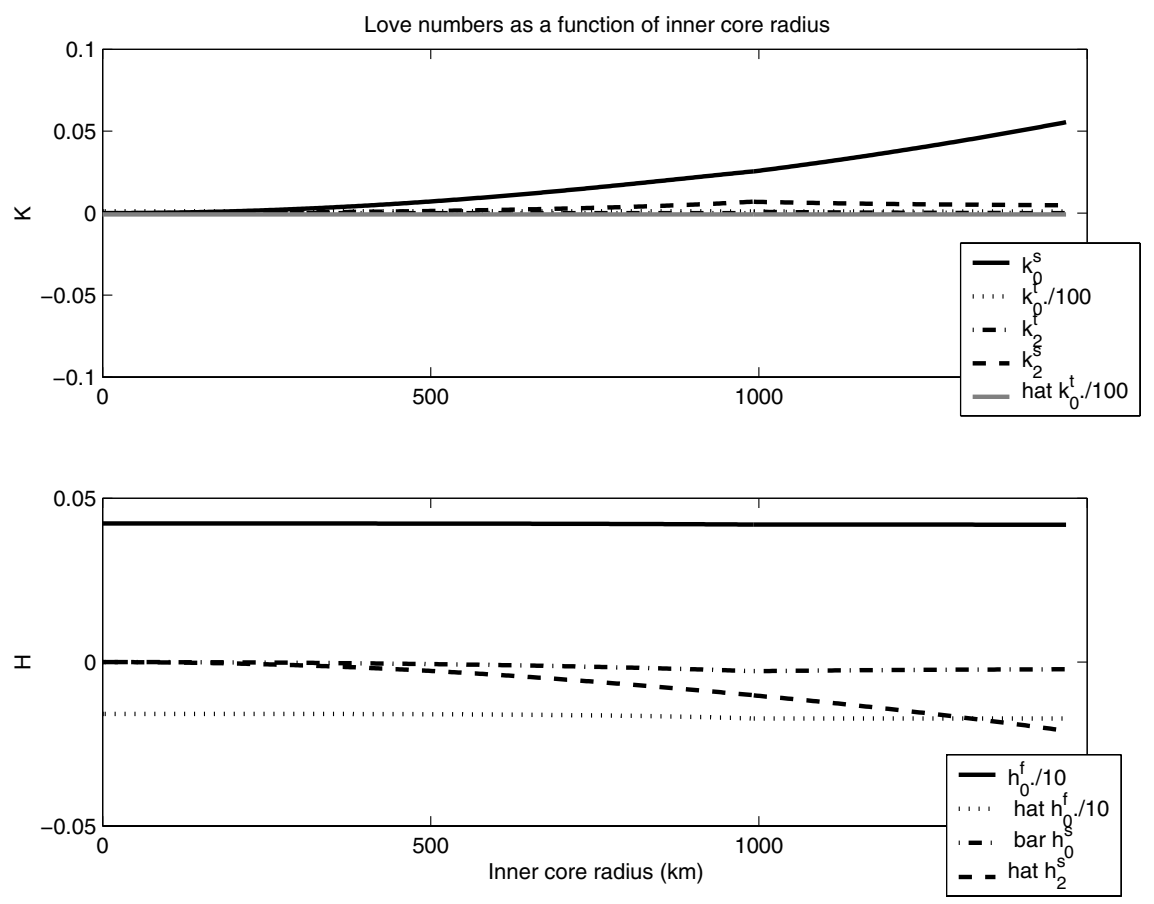

Fig. 2. Numerical values of the relevant Love numbers as a function of the inner core radius. The Love numbers are dimensionless quantities corresponding to a forcing equal to 1 .

Dehant et al. (2003), making reasonable hypotheses on the quality factor of the normal modes, computed the amplitudes of the normal modes for the particular evolutionary situation in which the inner core properties (radius, density, ...) are such that the resonance frequency of the ICW corresponds to one of the annual harmonics. For our models and a sulfur concentration of $14 \mathrm{wt} \%$, an inner core radius of $1260 \mathrm{~km}$ leads to an ICW period that is exactly equal to the annual period. The effect of the "annual ICW" conjunction on polar motion is largest if it corresponds to a large inner core moment of inertia, because the transfer function for polar motion is proportional to $A_{\mathrm{s}}$ (see Eq. (33)). Consequently, the lower the sulfur content of the core, the larger the inner core wobble will be at annual period.

In this study, we use the GCM of the Laboratoire Meteorologie Dynamique (LMD) (see Forget et al. 1999; Hourdin et al. 1995) to estimate the ice and atmosphere excitation function of Mars polar motion and to compute the polar motion for white noise excitation. The GCM computes the evolution of the system from a given initial state of the climate system (i.e. known pressure, temperature, and velocity field) by using well-known fluid mechanics and radiative transfer laws. Several physical parameterizations are needed in the dynamic model in order to properly handle the processes such as the radiative transfer, sub-grid scale dynamics, and condensation/sublimation of $\mathrm{CO}_{2}$. Some of the physical parameters in the model are adjusted to reproduce the surface pressure variations of Viking landers Hourdin et al. (1995).

In the present calculations, we assume a moderately dusty planet and use the Mars Global Surveyor Dust Scenario, which is based on recent spacecraft observations. The resulting $\mathrm{CO}_{2}$ cycle, and hence the atmospheric loading, is very sensitive to the albedo and the emissivity of the polar caps. We assume them to be 0.56 and 0.75 for the North pole and 0.58 and 0.85 for the South pole, respectively. Compared to previous studies, we use a slightly sharper surface resolution of $5.625^{\circ}$ by $3.83^{\circ}$ and a much higher temporal resolution with time steps of $2 \mathrm{~h}$, in order to include the effects of the diurnal cycle.

A simulation over 10 years is performed to properly assess the expected Chandler and Inner core wobble periods. The atmospheric excitation function, $\chi=\chi_{\mathrm{w}}+\chi_{\mathrm{p}}$, for the $x$ and $y$ components is of the same order of magnitude as reported by Van den Acker et al. (2002), but contains much more information since we consider the diurnal cycle instead of dealing only with a representative day per month over a year (see Fig. 3). The large oscillations starting approximately around day 400 correspond to the southern summer season when the atmospheric dust content is increased.

The Power Spectral Density (PSD) of the 10-year discrete time signals of $\chi_{x}$ and $\chi_{y}$ is computed using Welch's averaged, modified periodogram method (see Fig. 4). In the averaged spectra, the observed peaks for long periods (i.e. >100 days) correspond to the annual signal at 669 days and to its lower harmonics. The PSD of the atmospheric excitation for periods around 200 days (relevant for the Chandler wobble) is about $10^{-14}$, and for the inner core wobble $\left(500<P_{\text {ICW }}<\right.$ 1000) about $10^{-13}$. Note that these results depend largely on the method used for the PSD estimation. The applied Welch method generally yields the lowest PSD amplitudes. The highest estimates are obtained with autoregressive methods such as the Yule-Walker and Burg methods, which give PSD estimates on the order of $10^{-13}$ for the Chandler and $10^{-12}$ for the inner core wobble periods. 

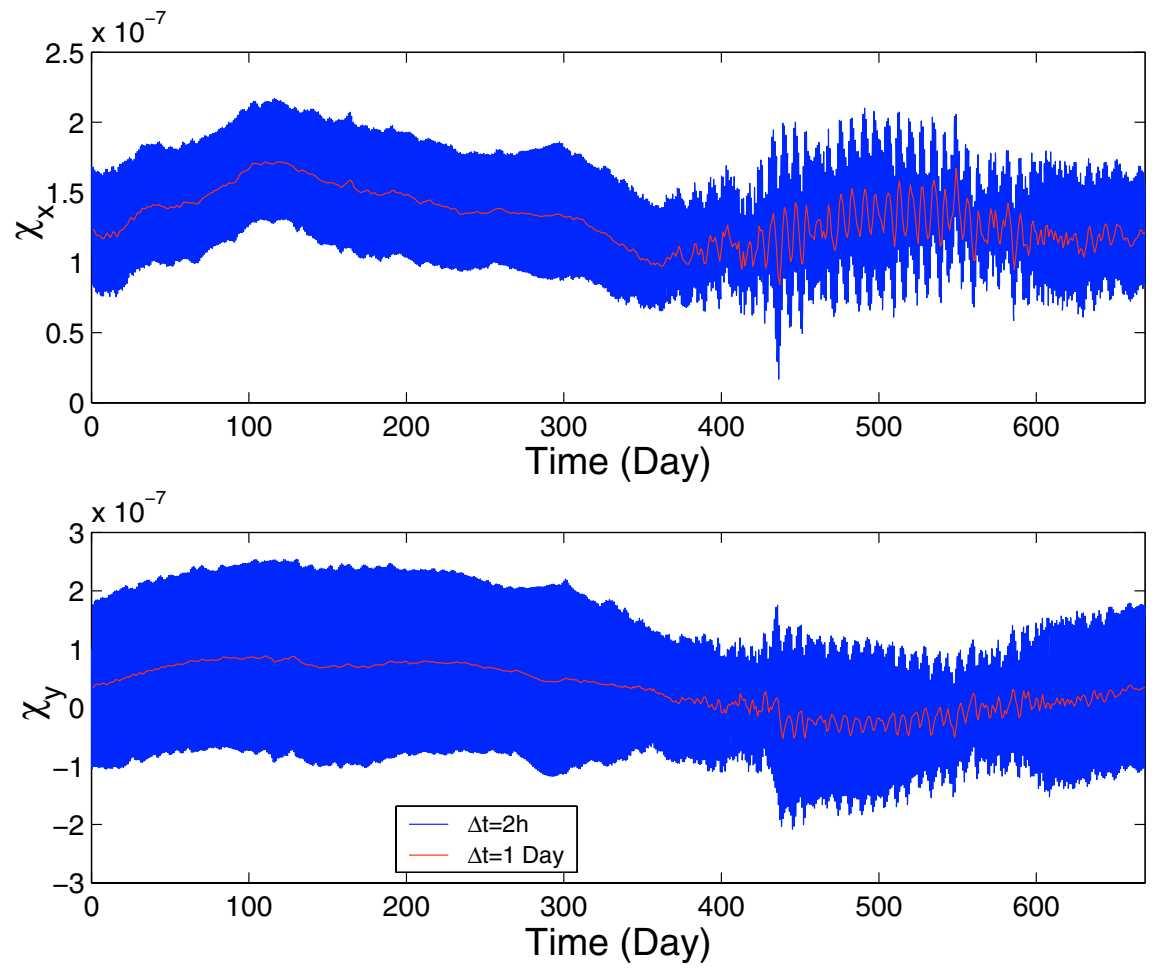

Fig. 3. Variation in atmospheric excitation functions over a Martian year.
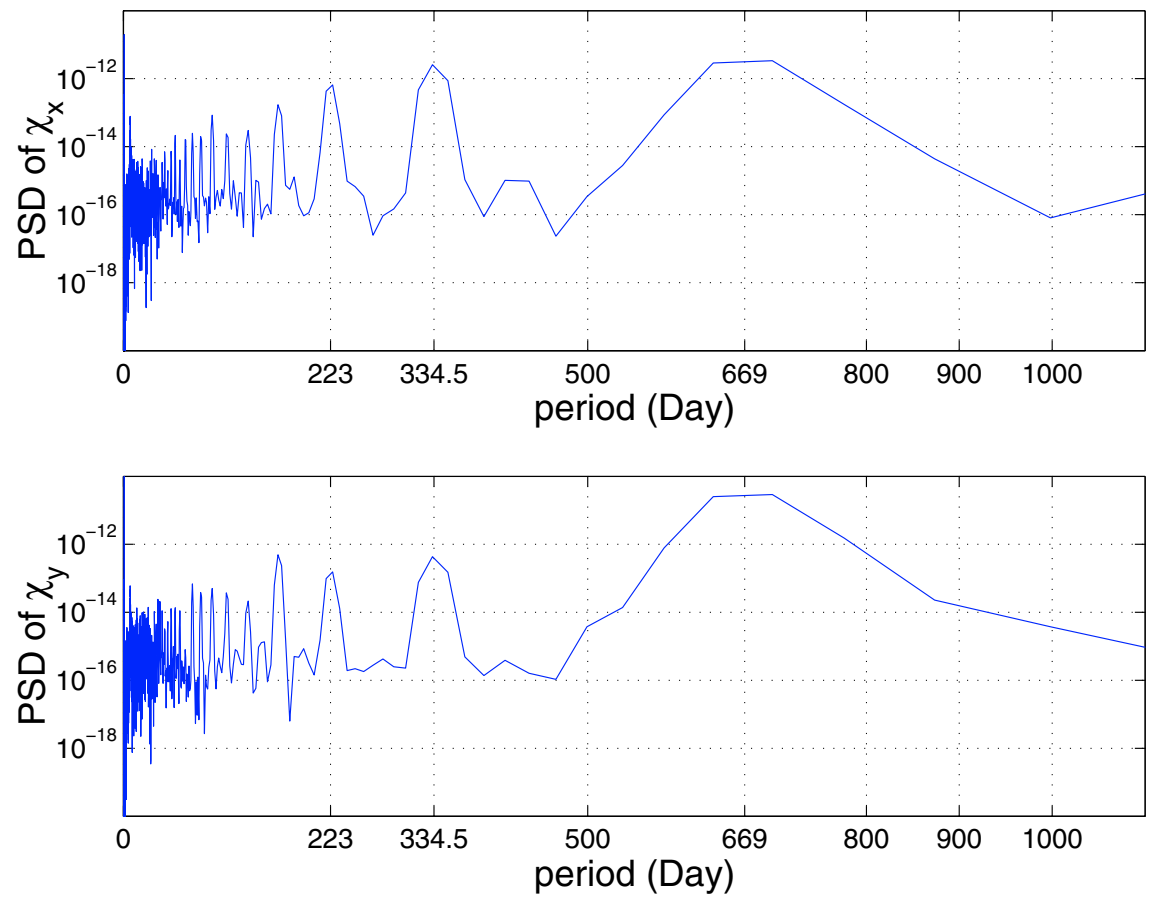

Fig. 4. Power spectral density estimates of $\chi_{x}$ and $\chi_{y}$.

The solution for the wobble $m$ as a function of the frequency and the forcing, as found in Sect. 3, has the form

$m=a_{0} f+\frac{a_{\mathrm{CW}}}{\sigma-\sigma_{\mathrm{CW}}} f+\frac{a_{\mathrm{ICW}}}{\sigma-\sigma_{\mathrm{ICW}}} f$,

where $a_{\mathrm{CW}}$ and $a_{\mathrm{ICW}}$ are the resonance strengths and depend on the physical cause of the excitation (pressure, wind, or external potential).
The polar motion of Mars $(p(\sigma))$ is given by (see for instance Gross 1992)

$p(\sigma)=\frac{1}{1+\frac{\sigma}{\Omega}} m(\sigma)$

The noise level of the excitation is characterized by its PSD. We transform the expression for polar motion as a function of the forcing into an expression for the PSD of polar motion as 

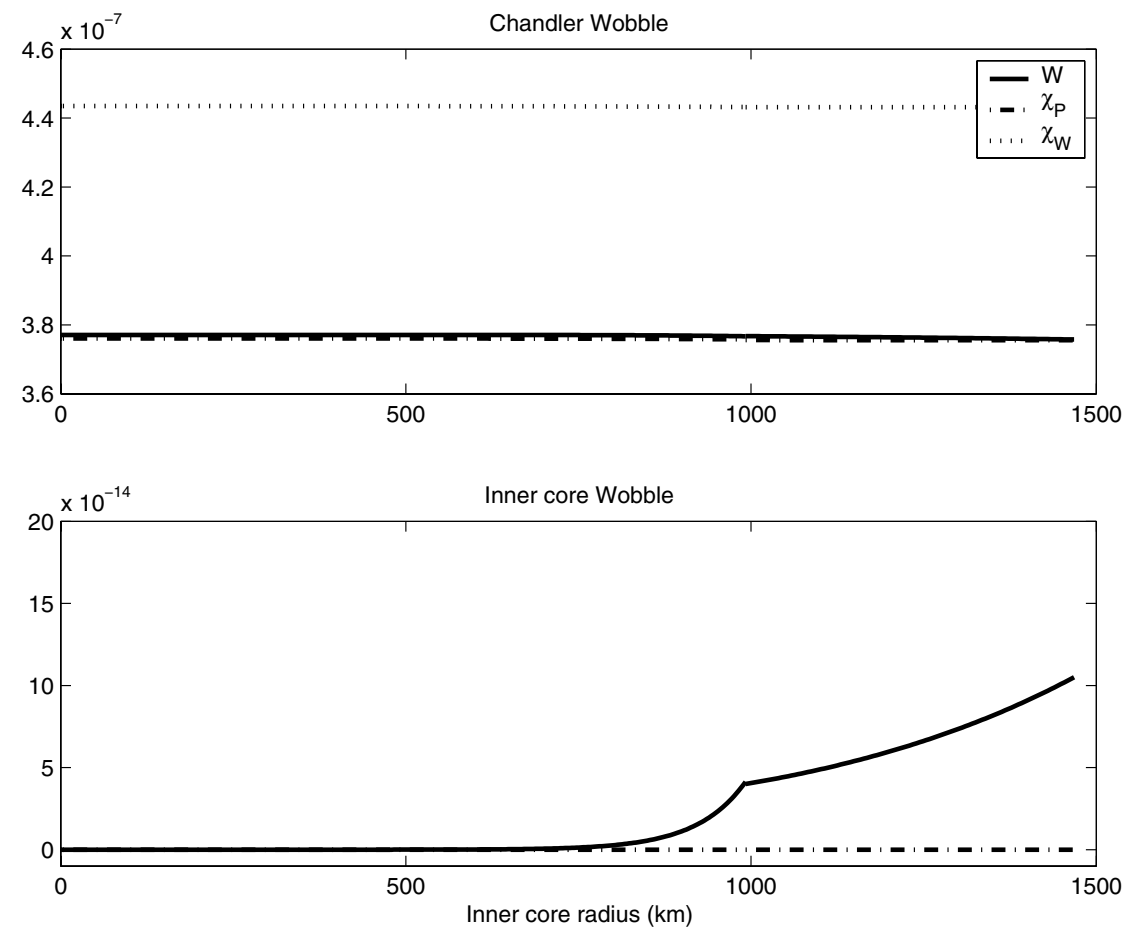

Fig. 5. Numerical value of the transfer function as a function of the inner core radius. The transfer functions are dimensionless quantities corresponding to the wobble in response to a forcing equal to 1 (in the case of the $\chi$ function).

a function of the PSD of the excitation functions $f$ associated with the noise. For a white noise we have

$f(\sigma) f^{*}(\sigma)=K$,

with $K$ independent on the frequency, and the symbol * denotes the complex conjugate.

In order to estimate the polar motion excited by a white noise, we use $P\left(\sigma_{\mathrm{R}}\right)$, the integral of the PSD over a given interval centered on $\sigma_{\mathrm{R}}$. For an arbitrarily small interval centered at the resonance frequency $\left(\sigma_{\mathrm{R}}\right)$, the power spectral density is computed as (Lambert 2003)

$P\left(\sigma_{\mathrm{R}}\right)=\int_{\sigma_{\mathrm{R}}-\delta \sigma_{\mathrm{R}}}^{\sigma_{\mathrm{R}}+\delta \sigma_{\mathrm{R}}} p(\sigma) p^{*}(\sigma) \mathrm{d} \sigma$.

In the case of a non-dissipative system, the amplitude of the normal mode would be infinite, which is not physical. Consequently, a small dissipation has to be taken into account, which can be done by adding an imaginary part to the resonance frequency (either the $\mathrm{CW}$ or the ICW frequency in this paper):

$\sigma_{\mathrm{R}}=\sigma_{\mathrm{r}}\left(1-\frac{\mathrm{i}}{2 Q_{\mathrm{R}}}\right)$

where $Q_{\mathrm{R}}$ is the quality factor of the resonance.

By integrating Eq. (37), we obtain

$$
\begin{aligned}
P\left(\sigma_{\mathrm{R}}\right)= & a_{\mathrm{R}}^{2} K \frac{-1}{2 \sigma_{\mathrm{r}} Q_{\mathrm{R}}}\left(1+4 Q_{\mathrm{R}}^{2}\right) \\
& {\left[\arctan \left(2 Q_{\mathrm{R}}\left(1-\frac{\sigma}{\sigma_{\mathrm{r}}}\right)\right)\right]_{\sigma_{\mathrm{R}}-\delta \sigma_{\mathrm{R}}}^{\sigma_{\mathrm{R}}+\delta \sigma_{\mathrm{R}}} } \\
\approx & 4 \frac{a_{\mathrm{R}}^{2} K}{\sigma_{\mathrm{r}}} Q_{\mathrm{R}} \arctan \left(\frac{2 Q_{\mathrm{R}}}{\sigma_{\mathrm{r}}} \delta \sigma_{\mathrm{R}}\right)
\end{aligned}
$$

where $a_{\mathrm{R}}$ is the resonance strength of the $\mathrm{CW}$ or the ICW, as defined in Eq. (34). By choosing $\delta \sigma_{\mathrm{R}}$ so that $99 \%$ of the mode energy is included in the interval, we have

$\delta \sigma_{\mathrm{R}}=31.83 \frac{\sigma_{\mathrm{r}}}{Q_{\mathrm{R}}}$.

By substituting this expression in Eq. (39), we then obtain

$P\left(\sigma_{\mathrm{R}}\right)=6.22 \mathrm{~K} \frac{a_{\mathrm{R}}^{2} Q_{\mathrm{R}}}{\sigma_{\mathrm{r}}}$.

In Fig. 5, we display the variable part $\left(\frac{a_{\mathrm{R}}^{2}}{\sigma_{\mathrm{r}}}\right)$ of $P\left(\sigma_{\mathrm{R}}\right)$ as a function of the inner core radius for the three excitations. Note that there is no $\chi_{\mathrm{w}}$ effect on the inner core wobble; indeed, the wind is associated with no change of the inertia tensor and creates no torque on the core. Additionally, the variation in the $\chi_{\mathrm{P}}$ effect is at the level of $10^{-19}$, which explains why it seems constant with the inner core radius in the graph.

The amplitude of polar motion resulting from the forcing of a normal mode by a white noise excitation is linear in the damping of the mode. For an undamped mode, the amplitude would be infinite. As a consequence, our results are very dependent on the quality factor used in the computation. For the Chandler wobble, we use values of the quality factor in the interval [80, 300]. The lower limit is a good compromise between the values obtained by Yoder (1982) and Smith et al. (1976). In a recent study, Bills et al. (2005) use the secular acceleration from the orbit of Phobos determined using astrometric observations and MOLA shadows cast by Phobos on the Martian surface. They estimate the value of $Q$ to be about 85 . The upper limit has been set to a high value (for the Earth, the Chandler Wobble $Q$ is at the level of 100) in order to explore a broader range of possibilities. In the case of the Inner Core Wobble, it is believed that the 


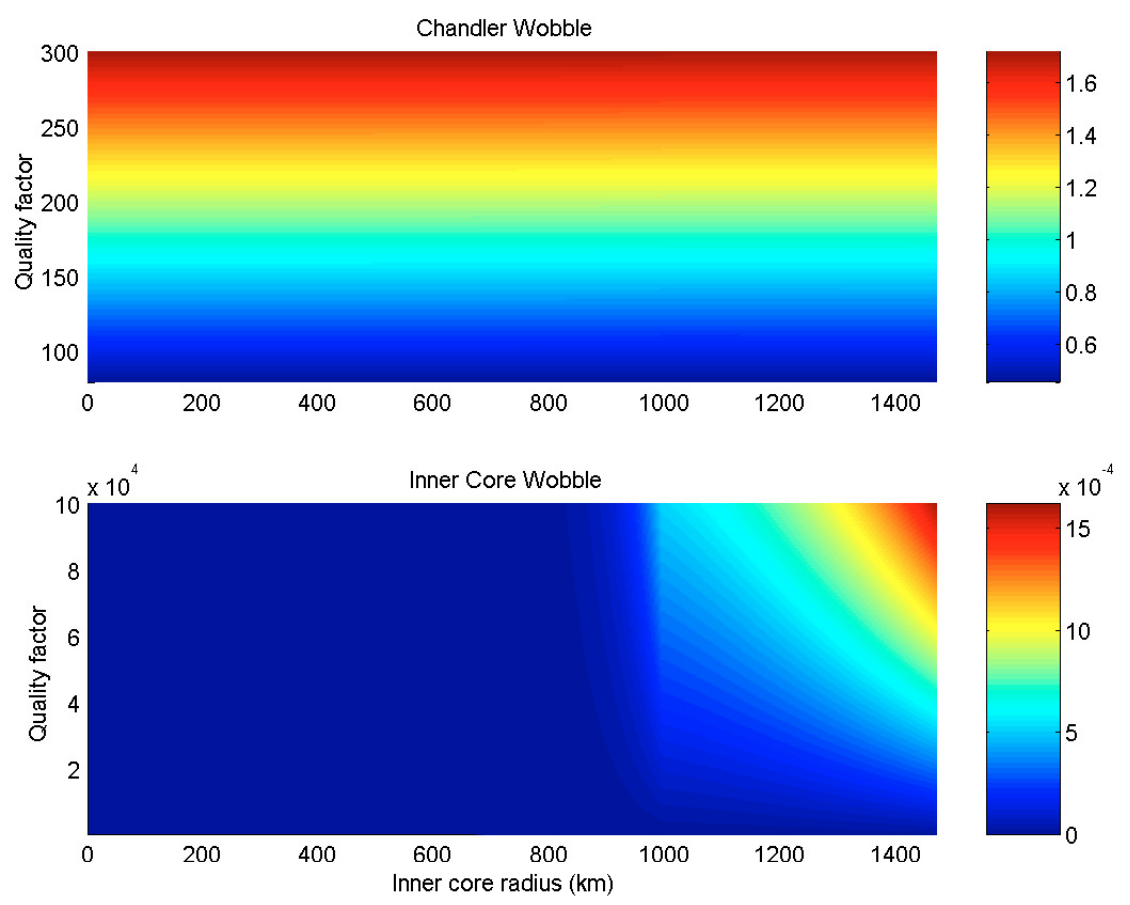

Fig. 6. Numerical value of the polar motion as a function of the quality factor and inner core radius. The amplitude is given in $\mathrm{m}$ for the Chandler wobble (top panel) and in mm for the Inner Core Wobble (bottom panel). The meaning of the colors is given in the right panels.

damping is very small due to the absence of a magnetic field, so we use $Q$ values between 600 (Earth value) and 100000 .

In Fig. 6, we show the amplitude (in meters and millimeters, respectively) of the Chandler wobble and of the Inner Core Wobble as a function of the quality factor and the inner core radius for values of the PSD of the atmospheric excitation at the level of several $10^{-15}$ for the Chandler wobble and of $10^{-14}$ for the inner core wobble. Even for the largest $Q$ values considered, the ICW amplitude is far below the expected observational precision of future space geodesy techniques (see for instance Dehant et al. 2003); it is thus very unlikely that this mode could be excited by the atmosphere at an observable level, even in the case of a very large value for $Q$ and a strong atmospheric excitation. Additionally, the amplitude and period of the CW resonance only weakly depend on the inner core radius, and the uncertainties on the other more relevant parameters will most probably hide the inner core signature in the CW signal.

\section{Effect of Marsquakes on polar motion}

From the paper by Ben-Menahem \& Israel (1970), it is a straightforward process to show that the change in the tensor of inertia is proportional to $\rho a^{2}$ for a homogeneous planet. The expressions also depend on the fault parameters, the quake moment, and the Poisson ratio parameter, which is the ratio between the transverse and longitidunal strains. For Mars, this ratio is most probably similar to the one of the Earth, as their compositions are comparable. According to Golombek et al. (1992), the maximum moment of a Marsquake would be at the level of $10^{16} \mathrm{Nm}$, which is very small compared to the largest Earth seismic moments (up to $10^{23} \mathrm{Nm}$ ). As $\rho a^{2}$ is 5 times smaller than for the Earth, and the moments are up to a factor $10^{7}$ smaller, we can expect a change of the inertia tensor of Mars that is about $10^{7}$ smaller for Mars than for the Earth, which could reach the centimeter level at best for one single event. As the Earth's moment of inertia is about 30 times larger than that of the Mars, the effects of Marsquakes on the rotation of Mars will be about $10^{5}$ times smaller than for the Earth. Consequently, even for the Chandler wobble, we can expect that it is impossible for a Marsquake to excite the polar motion to an observable extent.

\section{Conclusions}

Harmonic phenomena, such as the seasonal mass exhange between the atmosphere and the ice caps and periodic gravitational forcing from the Sun, can excite polar motion of Mars (Dehant et al. 2003; Defraigne et al. 2003). In this paper, we have studied two other excitation mechanisms of the polar motion of Mars: excitation by atmospheric stochastic forcing (or noise excitation) and sudden excitation by Marsquakes. In addition to the excitation of the Chandler wobble, we also studied the excitation of the Inner Core Wobble, which only exists if Mars has a solid inner core.

For harmonic excitation, as shown in Dehant et al. (2003), the ICW might be detected if its period is close enough to a harmonics of the seasonal cycle. This is the case because, at such periods, the power is strong and consistent in the atmospheric forcing, as well as in the gravitational forcing.

In this paper, we have shown that the Marsquake excitation is even smaller than is the case for the Earth. The main reason is the expected low energy of Marsquakes. Consequently, we mostly looked at the stochastic excitation that is due to the sublimation/condensation process of $\mathrm{CO}_{2}$, in order to estimate the level of polar motion induced, and the signature of the normal 
modes. We have developed the equation for computing the normal mode excitation by a random noise in the atmosphere and ice caps, estimated the excitation from output of the LMD atmospheric GCM, and computed the resulting $\mathrm{CW}$ to show that it is at an observable level, in case of a future mission dedicated to geodesy.

The sensitivity of the CW period and amplitude to the inner core radius is very small - at the level of 0.2 days for the period, and $0.1 \%$ for the amplitude (about 0.1 mas), under the hypotheses of this study - and is subcentimetric and consequently at the present limit of the observational precision on Earth's orientation, but not yet on Mars' orientation. Nevertheless, this level could be reached by increasing observational precision or by accumulating observations over the long term.

Our results also show how the amplitude of the CW excited by the atmospheric noise can be interpreted in terms of the inelasticity of the Martian mantle through the $\mathrm{CW}$ damping factor. Determining the damping of the Chandler wobble from observations is a challenging problem, as it is for the Earth. Nevertheless, complex statistical methods were developed for the Earth, in order to compensate for our lack of precise knowledge of the forcing at periods that do not correspond to harmonics of the seasonal cycles. The damping is then estimated from the observation of the mode itself under hypotheses of the type of forcing noise.

The amplitude of the ICW is larger for larger inner core and for larger $Q$. This is not surprising, as a larger inner core is associated with larger inertia and larger dynamic flattening, and a larger $Q$ is associated with less dissipation of the polar motion. We found a maximum value at the level of $2 \mu \mathrm{m}$. Such small amplitude of the ICW is far below the present level of observation precision. For instance, in the case of the NetLander mission (Harri et al. 1999; Dehant et al. 2004), which had a dedicated geodesy mission, a precision on the polar motion determination at the level of a few centimeters was expected (Barriot et al. 2001).

Analysis of polar motion, and in particular determination of its normal mode components, is promising because normal mode periods and amplitudes are directly related to the properties of the deep interior. The precision needed to get useful information is very demanding, and it requires improvement of the Martian rotation measurements. A first step could be deployment of a longstanding network of landers at the Martian surface.

Acknowledgements. The work of O.d.V. was financially supported by the Belgian Service Public fédéral de Programmation Politique scientifique. The work of O.K. was financially supported by the Belgian PRODEX program. The Laboratoire de météorologie dynamique is acknowledged for providing the atmospheric GCM. S. Lambert, C. Bizouard, and M. Greff-Lefftz are acknowledged for useful discussions. This work benefitted from the support of the European Community's Improving Human Potential Programme under contract RTN2-2001-00414, MAGE.

\section{References}

Barnes, R. T. H., Hide, R., White, A. A., \& Wilson, C. A. 1983, Proc. R. Soc. London, A 387, 31
Barriot, J.-P., Dehant, V., Folkner, W., et al. 2001, Adv. Space Res., 28(8), 1237

Ben-Menahem, A., \& Israel, M. 1970, Geophys. J. Roy. Astron. Soc., 19, 367

Bills, B. G., Neumann, G. A., Smith, D. E., \& Zuber, M. T. 2005, J. Geophys. Res., 110, E07004, DOI: 10.1029/2004JE002376

Cazenave, A., \& Balmino, G. 1981, Geophys. Res. Lett., 8, 245

Chao, B. F., \& Rubincam, D. P. 1990, J. Geophys. Res., 95, 14755

Dahlen, F. A. 1971, Geophys. J. Roy. Astron. Soc., 25, 157; Also: Dahlen, F. A. 1973, Geophys. J. Roy. Astron. Soc., 32, 203

Defraigne, P., de Viron, O., Dehant, V., Van Hoolst, T., \& Hourdin, F. 2000, J. Geophys. Res., 105(E10), 24563

Defraigne, P., Rivoldini, A., Van Hoolst, T., \& Dehant, V. 2003, J. Geophys. Res., 108(E12), 5128

Dehant, V., Hinderer, J., Legros, H., \& Lefftz, M. 1993, Phys. Earth Planet. Inter., 76, 259

Dehant, V., Van Hoolst, T., de Viron, O., et al. 2003, J. Geophys. Res., 108 (E12), 5127

Dehant, V., Lognonné, P., \& Sotin C. 2004, Planet. Space Sci., 52(11), 977

Dehant, V., de Viron, O., \& Greff-Lefftz, M. 2005, A\&A, 438, 1149

Forget, F., Hourdin, F., Fournier, R., et al. 1999, J. Geophys. Res., 104(E10), 24155

Greff-Lefftz, M., Legros, H., \& Dehant, V. 2000, Phys. Earth Planet. Inter., 122, 187

Greff-Lefftz, M., Dehant, V., \& Legros, H. 2002, Phys. Earth Planet. Inter., 129(1-2), 31

Gross, R. S. 1992, Geophys. J. Int., 109, 162

Golomek, M. P., Banerdt, W. B., Tanaka, K. L., \& Tralli, D. M. 1992, Nature, 248, 979

Harri, A.-M., Marsal, O., Lognonne, O., et al. 1999, Adv. Space Res., 23(11), 1915

Hourdin, F., Forget, F., \& Talagrand, O. 1995, J. Geophys. Res., 100(E3), 5501

Karatekin, O., Duron, J., Rosenblatt, P., et al. 2005, J. Geophys. Res., 110(E6), E06001

Karatekin, O., Van Hoolst, T., Tastet, J., de Viron, O., \& Dehant, V. 2005, Adv. Space Res., in press

Lambert, S. 2003, Analyse et modélisation de haute précision pour l'orientation de la Terre, Ph.D. Thesis, Observatoire de Paris, France, in French

Litvak, M. L., Mitrofanov, I. G., Kozyrev, A. S., et al. 2004, Sol. Sys. Res., 38, 167

Mathews, P. M., Buffett, B. A., Herring, T. A., \& Shapiro, I. I. 1991, J. Geophys. Res., 96(B5), 8219

Munk, W. H., \& McDonald, G. F. 1960, The Rotation of the Earth, a Geophysical discussion (Cambridge University Press), 323

Sanchez, B., Haberle, R., \& Schaeffer, J. 2004, J. Geophys. Res., 109, E08005

Sasao, T., \& Wahr, J. M. 1981, Geophys. J. Roy. Astron. Soc., 64, 729

Smith, J. C., \& Born, G. H. 1976, Icarus, 27, 51

Smith, D. E., Zuber, M. T., Haberle, R. M., Rowlands, D. D., \& Murphy, J. R. 1999, J. Geophys. Res., 104 (E1), 1885

Smith, D. E., Zuber, M. T., \& Neumann, G. A. 2001, Science, 294(5549), 2141

Sohl, F., \& Spohn, T. 1997, J. Geophys. Res., 102(1), 1613

Van den Acker, E., Van Hoolst, T., de Viron, O., et al. 2002, J. Geophys. Res., 107(E7), DOI: 10.1029/2000JE001539

Van Hoolst, T., Dehant, V., \& Defraigne, P. 2000, Planet. Space Sci., 48, 1145

Yoder, C. F. 1982, Icarus, 49, 327

Yoder, C. F., Konopliv, A. S., Yuan, D. N., Standish, E. M., \& Folkner, W. M. 2003, Science, 300, 299 